\title{
A NUMERICAL METHOD FOR THE SOLUTION OF PLANE CRACK PROBLEMS IN FINITE MEDIA
}

\author{
P. S. THEOCARIS and N. IOAKIMIDIS \\ Department of Theoretical and Applied Mechanics \\ The National Technical University of Athens \\ 5 K. Zografou Street, Zografou \\ Athens 624, Greece
}

(Recelved November 7, 1979)

ABSTRACT. A general method for the solution of plane isotropic elasticity crack problems inside a finite medium of arbitrary shape or an infinite medium with holes of arbitrary shape is presented. This method is based on the complex potential approach of plane elasticity problems due to Kolosov and Muskhelishvili [1] and makes no assumption on the way of loading of the cracks and of the other boundaries of the medium.

The whole problem is reduced to a complex singular integral equation along the cracks and the other boundaries and the values of the stress intensity factors at the crack tips may be evaluated directly and accurately from the numerical solution of this equation. An application of the method to a circular medium with a straight crack is also made.

KEY WORDS AND PHRASES. Singular integral equations, Plane curvilinear cracks, Numerical Solutions, stress intensity factors, complex potentials, Cauchy-type principal value integrals, Lobatto-Chebyshev numerical integration rule. 
1980 MATHEMATICS SUBJECT CLASSIFICATION CODES: $65 \times X, 65 R 05,73 \times X, 73.30,73.45$.

\section{INTRODUCTION.}

An efficient method for solving plane elasticity crack problems and estimating the stress intensity factors at crack tips is the method which reduces the problem to a Cauchy type singular integral equation (called in the sequel simply a singular integral equation) either by considering a curvilinear crack composed of a series of edge dislocations, or by using the complex potential technique of Kolosov and Muskhelishvili [1]. The first approach was developed in papers by Goldstein, Salganik and Savova [2-4]. These researchers reduced the problem of a curvilinear crack in an infinite medium to a system of two real Cauchy type singular integral equations along the crack by assuming that both its edges were loaded in the same way. The second approach was used by Lin'kov [5], who used the complex potentials $\phi(z)$ and $\psi(z)$ of Kolosov and Muskhelishvili [1] and assumed that the total force exerted on the curvilinear crack was vanished.

Panasyuk, Savruk and Datsyshin, in a series of papers the results of which were reviewed in [6], used the more practical complex potentials $\Phi(z)$ and $\Psi(z)$ of Kolosov and Muskhelishvili [1], but restricted their investigations to straight cracks, which are the most easy to solve. Finally, the present authors, using also the complex potentials $\Phi(z)$ and $\Psi(z)$, solved several general plane elasticity crack problems in infinite media [7-10] by relaxing any assumptions on the shape and loading mode of the cracks.

In this paper the previous methods were extended to cases of finite elastic media where the outer boundary or inside boundaries due to holes and other voids may interact with the cracks. The problem will be reduced to a complex singular integral equation along all boundaries of the plane elastic medium under consideration (the cracks included). For the numerical solution of this equation the numerical techniques developed by the authors in references [14] and [15] can 
be successfully used as already made in these references, as well as in references [10], [11]. Finally, an application will be made to the simple problem of a circular medium with a straight crack under constant pressure.

\section{AN INFINITE MEDIUM WITH HOLES AND CRACKS}

We consider an infinite isotropic elastic medium under generalized plane stress or plane strain conditions containing a system of arbitrary-shaped cracks $\mathrm{L}_{1 \mathrm{j}}$, as well as a system of holes $\mathrm{L}_{2 \mathrm{j}}$ (Fig.1). The material of the elastic medium is characterized by the elastic modulus $\mathrm{E}$ and the Poisson ratio $\nu$. The Poisson ratio $\nu$ is replaced by the constant $x$ [1] defined by $x=(3-v) /(1+\nu)$ for generalized plane stress conditions and by $x=3-4 \nu$ for plane strain conditions.

The loading of the medium is the most general consisting of loading the two faces of the cracks and the holes and of loading the plate at infinity. The loading on the cracks and the holes is characterized by its normal and shear components, $\sigma_{n}(t)$ and $\sigma_{t}(t)$, respectively (Fig. 1), where $t=x+i y$ denotes a generic point of the holes and the cracks in a Cartesian coordinate system 0xy. The loading distributions along the two faces $(+)$ and $(-)$ of the same crack may be different. Moreover, the positive directions along each crack (defining also the $(+)$ and $(-)$ edges of this crack) are defined arbitrarily, whereas the positive directions along the boundaries of the holes are considered counter clockwise. As regards the loading at infinity, it is assumed that we know the values of the principal stresses $N_{1}$ and $\mathrm{N}_{2}$, the angle formed between the direction of $\mathrm{N}_{1}$ and the $0 \mathrm{x}$-axis, as well as the value $\varepsilon_{\infty}$ of rotation at infinity. Since the value of $\varepsilon_{\infty}$ does not influence the stress field, it may be assumed having an arbitrary value, being equal to zero. Then the well-known constants $\Gamma$ and $\Gamma^{\prime}$ of Muskhelishvili [1], to which the complex potentials $\Phi(z)$ and $\Psi(z)(z=x+i y)$ tend at infinity, will be given by [1] :

$$
\lim _{z \rightarrow \infty}(z)=\Gamma=\frac{1}{4}\left(N_{1}+N_{2}\right)+i \frac{2 \mu}{x+1} \varepsilon_{\infty}, \mu=E /[2(1+\nu)],
$$




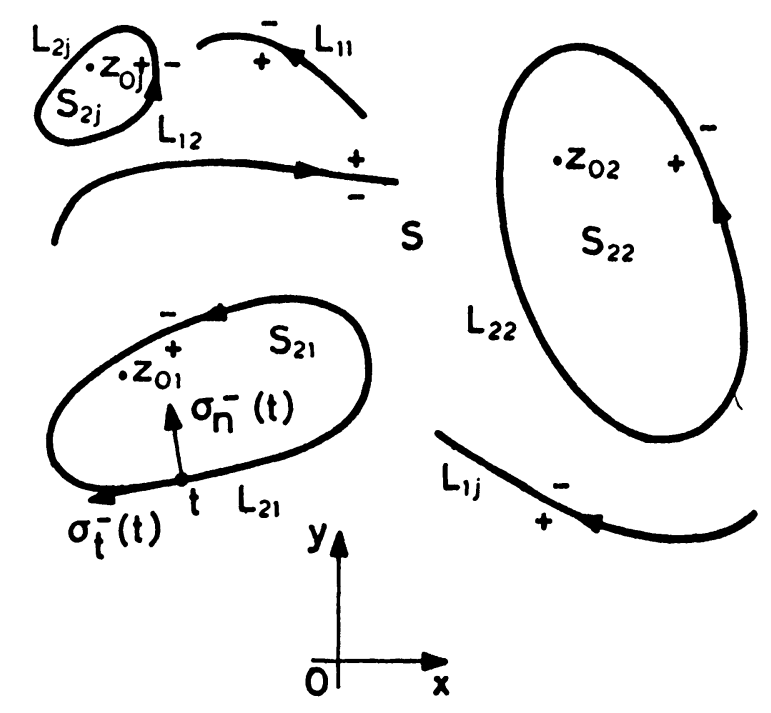

Fig. 1. An infinite plane medium with a set of holes and cracks.

$$
\lim _{z \rightarrow \ell} \Psi(z)=\Gamma^{\prime}=-\frac{1}{2}\left(\mathrm{~N}_{1}-\mathrm{N}_{2}\right) \exp (-2 i \alpha),
$$

where $\mu$ is the shear modulus of the elastic material.

The peculiarity of the problem under consideration, compared to other crack problems is the existence of holes of arbitrary shape inside the infinite medium which may be at any distance from the cracks.

Thus, the case solved in this paper is of great practical interest since not only the influence of the external boundary may be evaluated, but also the influence of voids and small discontinuities of the material close to the crack may be taken into account in defining the fracture mode of the substance. To maintain the general character of the formulation of the problem in terms of the complex potentials $\Phi(z)$ and $\Psi(z)$, we consider the holes $L_{2 j}$ as filled with inclusions of the same shape as the holes and consisting of the same material as 
the elastic medium. Along the boundaries $\mathrm{L}_{2 j}$ of these inclusions we assume no loading existing. Thus the boundaries $\mathrm{L}_{2 \mathrm{j}}$ of the holes can be considered as cracks loaded in a known way.

Now, following the developments of references [7-11], we determine the complex potentials $\Phi(z)$ and $\Psi(z)$ in terms of Cauchy type integrals :

$$
\begin{aligned}
& \Phi(z)=\frac{1}{2 \pi i} \int_{L} \frac{\varphi(\tau)}{\tau-z} d \tau+\Gamma, \\
& \Psi(z)=-\frac{1}{2 \pi i} \int_{L} \frac{\overline{\varphi(\tau)}-2 \overline{g(\tau)}}{\tau-z} \overline{d \tau}-\frac{1}{2 \pi i} \int_{L} \frac{\bar{\tau} \varphi(\tau)}{(\tau-z)^{2}} d \tau+\Gamma^{i},
\end{aligned}
$$

where $\mathrm{L}$ denotes both the cracks $\mathrm{L}_{1 j}$ and the boundaries $\mathrm{L}_{2 j}$ of the holes. In these equations the density $\varphi(t)$ is an unknown function of the points $t$ of $L$, whereas the function $q(t)$ is defined by :

$$
\begin{aligned}
& 2 q(t)=\left[\sigma_{n}^{+}(t)+i \sigma_{t}^{+}(t)\right]-\left[\sigma_{n}^{-}(t)+i \sigma_{t}^{-}(t)\right], \\
& 2 q(t)=-\left[\sigma_{n}^{-}(t)+i \sigma_{t}^{-}(t)\right]
\end{aligned}
$$

along the cracks $L_{1 j}$ and the boundaries $L_{2 j}$ of the holes respectively, because of the loading of the 'inclusions' already assumed. It can further be seen on the basis of the Plemelf formulae [1] that the boundary conditions along L are :

$$
2 \operatorname{Re} \Phi^{ \pm}(t)+\frac{d t}{d t}\left[-\Phi^{\prime} \Phi^{ \pm}(t)+\Psi^{ \pm}(t)\right]=\sigma_{n}^{ \pm}(t)-1 \sigma_{t}^{ \pm}(t), \frac{d t}{\overline{d t}} \equiv \frac{d t / d s}{\overline{d t} / d s},
$$

where $s$ is a variable denoting the arc-length. These conditions are satisfied if the density $\varphi(t)$ in equations (2) satisfies the complex singular integral equation :

$$
\begin{gathered}
\operatorname{Re}\left[\frac{1}{\pi i} \int_{L} \frac{\varphi(\tau)}{\tau-t} d t\right]-\frac{d t}{d t} \frac{1}{\pi i} \int_{L} \frac{\operatorname{Re}[(\bar{\tau}-\bar{t}) \varphi(\tau) d \tau]}{(\tau-t)^{2}}=\overline{p(t)}- \\
-2 \operatorname{Re} \Gamma-\frac{d t}{\frac{d t}{d t}}\left[\Gamma^{\prime}+\frac{1}{\pi i} \int_{L} \frac{\overline{q(\tau)}-\overline{d t}], \quad t \in L,}{\tau-t}\right]
\end{gathered}
$$

where the new function $p(t)$ is given by :

$$
2 p(t)=\left[\sigma_{n}^{+}(t)+i \sigma_{t}^{+}(t)\right]+\left[\sigma_{n}^{-}(t)+i \sigma_{t}^{-}(t)\right] \text {, }
$$




$$
2 p(t)=\sigma_{n}^{-}(t)+1 \sigma_{t}^{-}(t)
$$

along the cracks $L_{1 j}$ and the hole boundaries $L_{2 j}$ respectively.

Moreover, the conditions of single-valuedness of displacements around the cracks $\mathrm{L}_{1 \mathrm{j}}$ or the holes $\mathrm{L}_{2 \mathrm{j}}$ can be written as :

$$
\begin{aligned}
& \int_{L_{1 j}} \varphi(\tau) d \tau=\frac{2}{x+1} \int_{L_{1 j}} q(\tau) d \tau, j=1,2, \ldots, m_{1}, \\
& \int_{L_{2 j}} \varphi(\tau) d \tau=\frac{2}{x+1} \int_{L_{2 j}} q(\tau) d \tau,
\end{aligned}
$$

where $m_{1}$ and $m_{2}$ are the total numbers of cracks and holes respectively. Equations (7a) are analogous to those obtained in references [7-10] for cracks in an infinite isotropic elastic medium without holes. Equations ( $7 b$ ) coincide with the condition of single-valuedness of displacements for a finite medium or an infinite medium with a hole considered in reference [11].

The forms of equations (7a) and (7b) have no difference. In fact, under the present formulation the holes have been interpreted as contour-shaped cracks. Equations (7) supplement the singular integral equation (5) and have to be taken into account independently to obtain the correct results for the unknown density function $\varphi(t)$.

Although equations (5) and (7) are adequate to obtain a physically acceptable solution for the crack problem under consideration, yet they are not adequate for the single-valued determination of the unknown function $\varphi(t)$. This phenomenon is due to the presence of the holes, not the cracks, and was investigated in reference [11].

Briefly speaking, although we are sure, because of equation (4), about the vanishing of the total force and moment applied to each one of these inclusions, we have no guarantee that the complex potential $\Phi(z)$ is completely determined inside these inclusions, which psysically are completely separated from the 
elastic medium surrounding them. In fact, for finite media, a complex constant can be added to $\Phi(z)$ without any change in the stress field; only a rotation of the finite medium takes place.

In order to get rid of this arbitrariness we impose the further conditions : $\operatorname{Im} \Phi\left(z_{0 j}\right)=D_{j}, j=1,2, \ldots, m_{2}$,

where $z_{0 j}$ are points inside the inclusions $S_{2 j}$ and $D_{j}$ are arbitrary constants. These conditions define in a unique manner the imaginary constants just mentioned. Furthermore, because of equation (2a), valid in the whole elastic plane, equations (8) take the form :

$$
\operatorname{Im}\left[\frac{1}{2 \pi i} \int_{L} \frac{\varphi(\tau) d \tau}{\tau-z_{0 j}}\right]=E_{j}, j=1,2, \ldots, m_{2},
$$

where $\mathrm{E}_{\mathrm{j}}$ are also arbitrary constants.

Moreover, since the arbitrariness in the values of $\varphi(t)$ is restricted only along the hole boundaries $\mathrm{L}_{2 j}$, not along the cracks $\mathrm{L}_{1 j}$, we can replace equations (9) by :

$$
\operatorname{Im}\left[\frac{1}{2 \pi i} \int_{L_{2 j}} \frac{\varphi(\tau) d \tau}{\tau-z_{0 j}}\right]=0, j=1,2, \ldots, m_{2} .
$$

These conditions seem simpler to treat than conditions (9). However, neglecting these conditions does not lead to any erroneous results; just the values of $\varphi(t)$ along the contours $\mathrm{L}_{2 j}$ during the numerical solution of equations (5) and (7) are not convergent. On the contrary, the values of $\varphi(t)$ along the cracks $L_{1 j}$ are completely convergent.

Finally, it is convenient to incorporate conditions (7b) and (10) into the singular integral equation (5). This is because we thus impose the fulfilment of the boundary conditions at anyone of the collocation points used along the contours $\mathrm{L}_{2 j}$ during the numerical solution of the system of equations (5) and (7), by replacing the corresponding linear equation by the equation resulting from equation (7b) or (10) for the same contour $\mathrm{L}_{2 j}$. 
By taking into account the fact that equations (4) or (5) give no resultant force or moment on the inclusions $\mathrm{s}_{2 j}$, we introduce additional terms in equation (5) and neglect in the sequel equations ( $7 b)$ and (10). Thus we have :

$$
\begin{aligned}
\operatorname{Re}\left[\frac{1}{\pi I} \int_{L^{2}} \frac{\varphi(\tau)}{\tau-t} d \tau\right]-\frac{d t}{d t}\left\{\frac{1}{\pi I} \int_{L} \frac{\operatorname{Re}[(\tau-\bar{t}) \varphi(\tau) d \tau]}{(\tau-t)^{2}}+\delta(t)\left[\frac{C_{1 j}}{\left(t-z_{0 j}\right)^{2}} \operatorname{Re} \int_{L_{2 j}}\right.\right. \\
\left.\left.\frac{\varphi(\tau)}{\tau-z} d \tau+\frac{C_{2 j}}{t-z_{0 j}} \int_{L_{2 j}}\left[\varphi(\tau)-\frac{2}{x+1} q(\tau)\right] d \tau\right]\right\}=\overline{p(t)}-2 \operatorname{Re} \Gamma-\frac{d t}{\overline{d t}} \\
\\
{\left[\Gamma^{\prime}+\frac{1}{\pi i} \int_{L} \frac{\overline{q(\tau)}}{\tau-t}\right], \quad t \in L, }
\end{aligned}
$$

where :

$$
\begin{aligned}
& \delta(t)=1 \text { for } t \in L_{2 j}, j=1,2, \ldots, m_{2}, \delta(t)=0 \text { for } t \in L_{1 j}, \\
& j=1,2, \ldots, m_{1},
\end{aligned}
$$

and $C_{1 j}$ and $C_{2 j}$ are arbitrary constants, the first of which has a non-zero real part.

The values of these constants are assigned before the numerical solution of equation (11) together with conditions ( $7 a$ ) and, because of conditions ( $7 b$ ) and (10), they have theoretically no influence on the values of $\varphi(t)$ determined from the numerical solution of equations (11) and (7a).

We can also consider that $C_{1 j} \equiv 0$ and ignore the corresponding term in equation (11). Then this equation will have an infinite number of solutions, but all of them will be correct in the sense that they will satisfy all boundary and physical conditions of the problem and will give the correct values of the stress intensity factors at the crack tips.

However it is not permissible to neglect the term multiplied by $c_{2 j}$ in equation (11). This term substitutes the conditions of single-valuedness of displacements (7b) around the holes $\mathrm{L}_{2 \mathrm{j}}$ and should be taken into account. 


\section{A FINITE MEDIUM WITH A SYSTEM OF CRACKS}

The results of the previous section can also be directly generalized to the problem of a finite medium $S$ with a system of cracks $L_{j}(j=1,2, \ldots, m)$ as shown in Fig.2. The external boundary of the medium $S$ is denoted by $L_{0}$ and the boundary

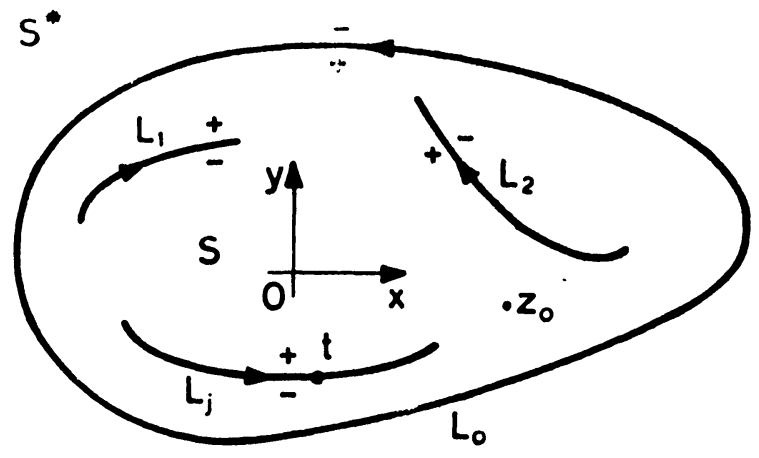

Fig. 2. A finite plane medium with a set of cracks.

conditions along $\mathrm{L}_{j}$ and $\mathrm{L}_{0}$ are assumed to be the same as those considered in the previous section. In this case we assume the infinite medium $\mathrm{S}^{*}$ outside $\mathrm{L}_{0}$ to be also occupied by the same isotropic elastic material as the medium S. Furthermore, we assume the loading distribution :

$$
p(t)=\sigma_{n}(t)+i \sigma_{t}(t)
$$

to act along $L_{0}$ on both media $S$ and $S^{*}$. This is contrary to what was made for the fictitious inclusions filling the holes in the previous section, which have been assumed unloaded, but it is preferable, since, in this way, we have :

$$
q(t)=0
$$

along $\mathrm{L}_{0}$ and equation (11) is simplified along this contour taking the form : 


$$
\begin{aligned}
& \operatorname{Re}\left[\frac{1}{\pi \dot{i}} \int_{L} \frac{\varphi(\tau)}{\tau-t} d \tau\right]-\frac{d t}{d t}\left\{\frac{1}{\pi \dot{i}} \int_{L} \frac{\operatorname{Re}[(\bar{\tau}-\bar{t}) \varphi(\tau) d \tau]}{(\tau-t)^{2}}+\right. \\
& \left.+\delta(t)\left[\frac{C_{1}}{\left(t-z_{0}\right)^{2}} \operatorname{Re} \int_{L_{0}} \frac{\varphi(\tau)}{\tau-z_{0}} d \tau+\frac{C_{2}}{t-z_{0}} \int_{L_{0}} \varphi(\tau) d \tau\right]\right\}= \\
& =\overline{p(t)}-\frac{d t}{d t} \frac{1}{\pi i} \int_{L} \frac{\overline{q(t)}}{\tau-t \tau}, t \in L,
\end{aligned}
$$

where $\mathrm{L}_{0}$ denotes the contour surrounding the elastic medium, $\mathrm{z}_{0}$ is a point inside the elastic medium (not belonging to $L_{0}$ or the cracks $L_{j}$ ), $L$ denotes all the boundaries of the medium (the cracks included), that is $\mathrm{L}=\mathrm{L}_{0} \mathrm{UL}_{1} \mathrm{UL}_{2} \mathrm{U}$. . UL $\mathrm{U}_{\mathrm{m}}$ and the function $\delta(t)$ is defined as :

$$
\delta(t)=1 \text { for } t \in L_{0}, \delta(t)=0 \text { for } t \in L \cap L_{0} \text {. }
$$

Moreover, the function $\mathrm{p}(\mathrm{t})$ is defined by equation (6a) along the cracks $\mathrm{L}_{j}$ and by equation (13) along the contour $L_{0}$. Also the function $q(t)$ is defined by equation (3a) along the cracks and vanishes, in accordance with equation (14), along $L_{0}$. The conditions ( $7 a)$ of single-valuedness of displacements along the cracks have also to be satisfied when solving equation (15) independently of this equation, that is :

$$
\int_{L_{j}} \varphi(\tau) d \tau=\frac{2}{x+1} \int_{L_{j}} q(\tau) d \tau, j=1,2, \ldots, m .
$$

The complex potentials $\Phi(z)$ and $\Psi(z)$ remain given by equations (2) without the presence of the constants $\Gamma$ and $\Gamma^{\prime}$, which have no meaning in the case of a finite isotropic elastic medium.

It is now necessary to show that equation (15) ensures the single-valuedness of the unknown density function $\varphi(t)$ along $L_{0}$, that is :

$$
\int_{\mathrm{L}_{0}} \varphi(\tau) \mathrm{d} \tau=0
$$

which is equivalent to the condition of single-valuedness of displacements along 
the fictitious crack $L_{0}$ formed between the real medium $S$ and the fictitious medium $\mathrm{S}^{*}$, as well as the single-valuedness of the complex potential $\Phi(z)$, expressed here as :

$$
\operatorname{Re} \int_{L_{0}} \frac{\varphi(\tau)}{\tau-z_{0}} d \tau=0,
$$

Equation (19) is sufficient, so that no arbitrary purely imaginary constant can be added to the complex potential $\Phi(z)$ rendering it multivalued, since such a constant would change the value of the integral in equation (19) because of the formula [1] :

$$
\frac{1}{2 \pi i} \int_{L_{0}} \frac{C}{\tau-z_{0}} d \tau=C
$$

if $L_{0}$ is a contour surrounding a finite simply-connected medium $S$ and $z_{0} \in S$ as happens in this case.

It is worthwhile mentioning that we may ignore both conditions (18) and (19) together with the term multiplied by $\delta(t)$ in equation (15). Then, this equation supplemented by conditions (17) will have not a unique solution, but this is not of much importance since any approximate solution obtained by solving this modified form of equation (15) will satisfy this equation that is the boundary conditions of our problem. This means that if, for example, we consider the approximate values for the stress intensity factors at the crack tips obtained by solving approximately equation (15), they will converge to their correct values. Yet, there are two reasons for which one wants that equation (15) should have a unique solution: The first is that is customary in plane elasticity problems to make use of singular integral equations possessing a unique solution: and not an infinity of solutions as it would be the case, if the term multiplied by $\delta(t)$ were ignored. However, a strict proof of the uniqueness of solution of equation (15) together with conditions (17) is beyond the aims of this paper and not at all straight forward. 
The second reason for which the complete form of equation (15) has to be used is that, in this way, the numerical results for $\varphi(t)$ along $L_{0}$ will be convergent to specific values along $L_{0}$ and not to floating ones. This is sometimes useful in order to estimate the efficiency of the numerical technique used.

We will show now that the term multiplied by $\delta(t)$ in equation (15) forces the satisfaction of conditions (18) and (19). If the medium $S$ contained no cracks, this statement would be evident in view of the developments of reference [11]. But in the present case this is not evident especially in the cases when there exist resultant forces and moments exerted on the cracks $L_{j}$. The results of reference [11] have to be appropriately generalized. Thus, by taking into account the boundary conditions (4), as well as equations (14) and (16), we can write the singular integral equation (15) as :

$$
\begin{gathered}
\Phi(t)+\overline{\Phi(t)}+\frac{d t}{\frac{d t}{t}}\left[\overline{\Phi^{\prime}}(t)+\Psi(t)\right]-\frac{d t}{d t}\left[\frac{C_{1}}{\left(t-z_{0}\right)^{2}} \operatorname{Re} \int_{L^{\tau-z_{0}}} \frac{\varphi(\tau)}{\tau t}+\right. \\
\left.+\frac{C_{2}}{t-z_{0}} \int_{L_{0}} \varphi(\tau) d \tau\right]=\overline{p(t)}, t \in L_{0} .
\end{gathered}
$$

We will show that both conditions (18) and (19) will be satisfied when equation (15), together with conditions (17), is solved, or, equivalently, that any solution of equation (21) satisfies the boundary condition :

$$
\Phi(t)+\overline{\Phi(t)}+\frac{d t}{d t}\left[\bar{t} \Phi^{\prime}(t)+\Psi(t)\right]=\overline{p(t)}, \quad t \in L_{0} .
$$

In equations (21) and (22) and in the sequel the symbols $\Phi(t), \Phi^{\prime}(t)$ and $\Psi(t)$ denote the boundary values of the functions $\Phi(z), \Phi^{\prime}(z)$ and $\Psi(z)$ as $z$ lies inside $S$ and approaches the point $t$ of $\mathrm{L}_{0}$.

If we denote by $(X, Y)$ the resultant force exerted along the whole system of cracks $L_{c}\left(L_{c}=L_{1} U L_{2} U \ldots U L_{m}\right)$, then, because of equation (3a), we have :

$$
2 \int_{L_{c}} q(\tau) d \tau=i P, P=X+i Y .
$$


Moreover, since the medium $S$ is assumed in equilibrium, the resultant force exerted along $\mathrm{L}_{0}$ should be opposite to $(\mathrm{X}, \mathrm{Y})$. Then, because of equation (13), we find :

$$
\int_{L_{0}} p(\tau) d \tau=-i P
$$

By taking also into account that :

$$
\begin{aligned}
& \int_{L_{0}} \Phi(\tau) \mathrm{d} \tau=-\int_{L_{c}}\left[\Phi^{+}(\tau)-\Phi^{-}(\tau)\right] \mathrm{d} \tau, \\
& \int_{L_{0}} \Psi(\tau) \mathrm{d} \tau=-\int_{L_{c}}\left[\Psi^{+}(\tau)-\Psi^{-}(\tau)\right] \mathrm{d} \tau,
\end{aligned}
$$

as well as equation (2) yielding, by application of the first formula of P1emelj [1]:

$$
\begin{aligned}
& \Phi^{+}(t)-\Phi^{-}(t)=\varphi(t), \\
& \Psi^{+}(t)-\Psi^{-}(t)=\frac{\overline{\mathrm{dt}}}{\overline{\mathrm{dt}}}[2 \overline{q(t)}-\overline{\varphi(t)}]-\frac{\mathrm{d}}{\mathrm{dt}}[\bar{t} \varphi(t)],
\end{aligned}
$$

and equations (17) and (23), we can find that :

$$
\begin{aligned}
& \int_{L_{0}} \Phi(\tau) \mathrm{d} \tau=-\frac{i}{x+1} P, \\
& \int_{L_{0}} \Psi(\tau) \mathrm{d} \tau=\frac{i x}{x+1} \bar{P} .
\end{aligned}
$$

By multiplying both sides of equation (22) by $\overline{\mathrm{dt}}$, integrating along $\mathrm{L}_{0}$ and taking into account equations (24) and (27), as well as the fact that :

$$
\int_{L_{0}} d[\bar{\tau} \Phi(\tau)]=0,
$$

we derive that both sides of the resulting equation are identically equal to $i \vec{P}$. This means that, if the same procedure is used for equation (21), then, because of equation (20) too, we must have :

$$
C_{2} \int_{L_{0}} \varphi(\tau) \mathrm{d} \tau=0
$$


or since $C_{2} \neq 0$ by assumption, condition (18) is satisfied.

In another wording, the addition of the term multiplied by the constant $\mathrm{C}_{2}$ to the boundary condition (22) to obtain the boundary condition (21), assures the satisfaction of the boundary condition (22).

Furthermore, if we denote by $\left(M_{x}, M_{y}\right)$ the resultant moment of the forces exerted on the system of cracks $L_{c}$, then the opposite moment should result which is exerted on the boundary $L_{0}$ by the loading applied along this boundary since the medium $\mathrm{S}$ was assumed in the state of equilibrium. In this case, it is easy to see that :

$$
\operatorname{Re} \int_{L_{0}} p(\tau) \bar{\tau} d \tau=-M, M=M_{x}+i M_{y},
$$

Furthermore, by taking into account equation (26b), it is possible to show that :

$$
\operatorname{Re} \int_{L_{c}} \Psi(\tau) \bar{\tau} \mathrm{d} \tau=-M
$$

and, further, to prove that the term multiplied by the constant $\mathrm{C}_{1}$ in equation (21) should vanish.

Hence, the addition of this term to the boundary condition (22) does not prevent this condition from being satisfied. In this way, the proof that equation (15), equivalent to (21), assures the fulfilment of both conditions (18) and (19) and the satisfaction of the boundary condition (22) along $\mathrm{L}_{0}$ has been completed.

\section{THE NUMERICAL TECHNIQUE}

The numerical solution of the singular integral equations derived in this paper can be achieved by reducing such an equation to a system of linear equations. This can be achieved by approximating the integrals through the use of appropriate numerical integration rules and applying the resulting equation at appropriately selected collocation points.

To illustrate this technique, we consider the simple problem of a finite isotropic elastic medium $S$ surrounded by a smoath contour $\mathrm{L}_{0}$ and containing a 
simple smooth crack $L_{c}$. In this case we assume that we know the parametric equations at $\mathrm{L}_{0}$ :

$$
z_{0}=z_{0}+i y_{0}=x_{0}\left(s_{0}\right)+i y_{0}\left(s_{0}\right)=z_{0}\left(s_{0}\right)
$$

and at $\mathrm{L}_{\mathrm{c}}$ :

$$
z_{c}=x_{c}+i y_{c}=x_{c}\left(s_{c}\right)+i y_{c}\left(s_{c}\right)=z_{c}\left(s_{c}\right),
$$

where $s_{0}$ and $s_{c}$ are real variables varying along $L_{0}$ and $L_{c}$ respectively. Without loss of generality we can assume that $s_{0}$ and $s_{c}$ vary in the intervals $[0,2 \pi]$ and $[-1,1]$ respectively. In the opposite case this can easily be achieved through linear variable tranformation of the from :

$$
\mathrm{s}^{-}=\mathrm{as}+\mathrm{b}
$$

where $\mathrm{a}$ and $\mathrm{b}$ are appropriate constants.

Moreover, we assume to know the normal and shear loading components along $\mathrm{L}_{0}$ and $\mathrm{L}_{\mathrm{c}}$ :

$$
\begin{array}{ll}
p_{0}(t)=\sigma_{n}\left(s_{0}\right)+i \sigma_{t}\left(s_{0}\right), & t \in L_{0}, \\
p_{c}(t)=\sigma_{n}\left(s_{c}\right)+i \sigma_{t}\left(s_{c}\right), & T \in L_{c},
\end{array}
$$

assuming, for simplicity, that the same loading distribution $\mathrm{p}_{c}(t)$ is exerted along both edges $(+)$ an'd $(-)$ of the crack $L_{c}$. Finally, the constant $z_{0}$ in equation (15) is assumed to have an appropriate value $z_{0}=c$ such that the point $z_{0}$ lies inside $S$, but not on the crack $L_{c}$. Similarly, the constants $C_{1}$ and $C_{2}$ in this equation are assumed having concrete values.

For numerical integrations along the contour $\mathrm{L}_{0}$ we can use the well-known trapezoidal rule with $\mathrm{n}_{0}$ abscissae. By defining the abscissae $\tau_{0 i}$ and the collocation points $t_{0 k}$ along $\mathrm{L}_{0}$ by :

$$
\begin{aligned}
& { }^{\tau_{0 i}}=z_{0}\left(\sigma_{0 i}\right), \sigma_{0 i}=2 \pi i / n_{0}+\vartheta_{0}, i=1,2, \ldots, n_{0} \\
& t_{0 k}=z_{0}\left(s_{0 k}\right), s_{0 k}=(2 k-1) \pi / n_{0}+\vartheta_{0}, k=1,2, \ldots, n_{0}
\end{aligned}
$$


where $\vartheta_{0}$ is an arbitrary constant, we have :

$$
\int_{L_{0}} h_{0}\left(\tau, t_{0 k}\right) d \tau \simeq \sum_{i=1}^{n_{0}} A_{0 i} h_{0}\left(\tau_{0 i}, t_{0 k}\right),
$$

the weights $\mathrm{A}_{01}$ determined by :

$$
A_{0 i}=2 \pi z_{0}^{-}\left(\sigma_{0 i}\right) / n_{0}, \quad i=1,2, \ldots, n_{0} .
$$

the numerical integration rule (39) is valid both for regular and Cauchy-type integrals provided that the points $t_{0 k}$ are determined by equation (38).

Moreover, for the numerical integrations along the crack $L_{c}$ we can use the Lobatto-Chebyshev numerical integration rule [12] with $n_{c}$ abscissae. By defining the abscissae $\tau_{c i}$ and the collocation points $t_{c k}$ along $L_{c}$ by :

$$
\begin{aligned}
& \tau_{c i}=z_{c}\left(\sigma_{c i}\right), \sigma_{c i}=\cos \left[\pi(i-1) / n_{c}-1\right], i=1,2, \ldots, n_{c}, \\
& t_{c k}=z_{c}\left(s_{c k}\right), s_{c k}=\cos \left[\pi(k-0.5) /\left(n_{c}-1\right)\right], k=1,2, \ldots, n_{c}-1,
\end{aligned}
$$

we have :

$$
\int_{L_{c}}\left(1-s_{c}^{2}\right)^{-\frac{1}{2}} h_{c}\left(\tau, t_{c k}\right) d \tau \simeq \sum_{i=1}^{n_{c}} A_{c i} h_{c}\left(\tau_{c i}, t_{c k}\right),
$$

the weights $A_{c i}$ determined by :

$$
\begin{aligned}
& A_{c i}=\pi \lambda_{i} z_{c}^{-}\left(\sigma_{c i}\right) /\left(n_{c}-1\right), \\
& \lambda_{i}=1, i=2,3, \ldots, n_{c}-1, \lambda_{i}=\frac{1}{2}, i=1, n_{c} .
\end{aligned}
$$

In this way, there exist $\left(\mathrm{n}_{0}+\mathrm{n}_{c}\right)$ complex unknowns in equations (15) and (17), the values of $\varphi(t)$ on $L_{0}$ and $x(t)=\varphi(t)$. $\left(1-s_{c}^{2}\right)^{\frac{1}{2}}$ on $L_{c}$ at the abscissae used $\tau_{01}$ and $\tau_{c i}$ along $L_{0}$ and $L_{c}$ respectively, or, equivalently, $2\left(n_{0}+n_{c}\right)$ real unknowns in these equations.

Furthermore, we obtain $\mathrm{n}_{0}$ complex linear equations by applying equation (15) at the collocation points $t_{0 k}$ along $L_{0}$ and $\left(n_{c}-1\right)$ complex linear equations by 
applying the same equation at the collocation points $t_{c k}$ along $L_{c}$.

One more linear equation is obtained from the condition of single-valuedness of displacements (17). In this way, we have finally $\left(\mathrm{n}_{0}+\mathrm{n}_{c}\right)$ complex linear equations, or $2\left(n_{0}+n_{c}\right)$ real linear equations with an equal number of unknowns. By solving this system of equations we obtain the approximate values of the unknown function $\varphi(t)$ at the abscissae $\tau_{0 i}$ along $L_{0}$, as well as the values of $\chi(t)=\varphi(t) \cdot\left(1-s_{c}^{2}\right)^{\frac{1}{2}}$ at the abscissae $\tau_{c i}$ along $L_{c} \cdot$.

We can also mention that it is very advantageous to use complex arithmetic in the computer for the evaluation of all required quantities and especially the matrix of coefficients of the system of linear equations to be solved. Moreover, it is evident that the factor $d t / \overline{d t}$ in equation (15) can easily be computed at a collocation point $\mathrm{t}_{\mathrm{Ok}}$ of $\mathrm{L}_{0}$ or $\mathrm{t}_{\mathrm{ck}}$ of $\mathrm{L}_{\mathrm{c}}$ as :

$$
\begin{gathered}
{[\mathrm{dt} / \overline{\mathrm{dt}}]_{t=t_{0 k}}=z^{-}\left(s_{0 k}\right) / \overline{z^{-}\left(s_{0 k}\right)},[d t / \overline{d t}]_{t=t_{c k}}=} \\
z^{-}\left(s_{0 k}\right) / \overline{z^{-}\left(s_{c k}\right)} .
\end{gathered}
$$

Finally, the complex stress intensity factors $\mathrm{K}_{A}$ and $\mathrm{K}_{\mathrm{B}}$ at the tips of the crack $L_{c^{-}}$corresponding to $s_{c}=-1$ and $s_{c}=+1$, can easily be computed if one takes into account the formula :

$$
K=2 \cdot 2^{\frac{1}{2}} \exp \left(-\frac{1 \vartheta_{c}}{2}\right) \lim _{z \rightarrow c}\left[(z-c)^{\frac{1}{2}} \Phi(z)\right],
$$

where $c$ is the value of the complex variable $z=x+i y$ corresponding to the crack tip under consideration and $\vartheta_{c}$ the angle between the $0 x$-axis and the tangent to the crack at the crack tip $z=c$ in the direction of extension of the crack. Then we can find that :

$$
\begin{aligned}
& K_{A}=K_{I A}-1 K_{I I A}=i\left|z_{c}^{-}(-1)\right|^{\frac{1}{2}} \chi\left(z_{c}(-1)\right), \\
& K_{B}=K_{I B}-i K_{I I B}=-1\left|z_{c}^{-}(+1)\right|^{\frac{1}{2}} \chi\left(z_{c}(+1)\right) .
\end{aligned}
$$


Since the Lobatto-Chebyshev numerical integration rule for the numerical integrations along the crack $\mathrm{L}_{0}$ contains among the abscissae used, equations (41), the end-points $\sigma_{c}= \pm 1$ of the integration interval, the evaluation of the stress intensity factors at the crack tips, by using equations (47), is straight-forward. Moreover, we can mention that it is also possible to treat the cases of cracks of complicated shape like branched cracks or cruciform cracks by applying techniques presented in reference [14] and references [13] and [15] respectively. Similarly, it is possible to apply the present technique to the case of edge cracks, as made in reference [16] for the case of a crack terminating at the boundary of a half-plane. In this case, no condition of single-valuedness of displacements has to be taken into account along the cracks. Furthermore, in the case when a crack tip approaches another crack tip or a boundary of the elastic medium, the modification of the Lobatto-Chebyshev method proposed in reference [17] can be successfully used. Also in the case of semi-infinite straight or curvilinear cracks, the Lobatto-Chebyshev method of numerical integration along the cracks should be replaced by the methods proposed in references [18] and [19]. Several more numerical techniques for the solution of singular integral equations, which may be applied to the solution of the singular integral derived in this paper, are reviewed in reference [20].

\section{AN APPLICATION}

As an application we consider the problem of a circular plane isotropic elastic medium with a straight crack along one of its diameters assumed for simplicity to coincide with the Ox-axis, as shown in Fig.3. The crack is assumed to be loaded by a pressure of constant intensity $\sigma$, whereas the circumference of the circular medium is assumed unloaded. The radius of the circular medium is denoted by $R$, the length of the crack by $2 a$ and the distance of the middle-point $M$ of the crack from the centre 0 of the circle by $d$. 


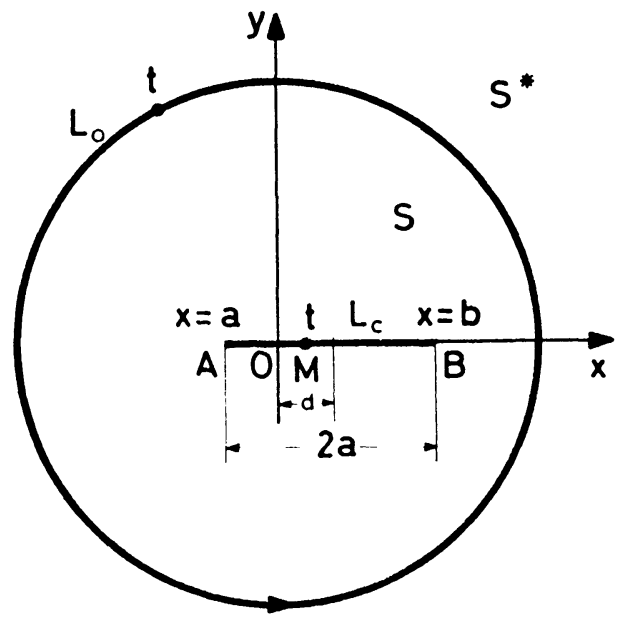

Fig. 3. A circular disc with a crack along one of its diameters.

Then, in accordance with the notation of the previous section we will have :

$$
z_{0}(s)=\operatorname{Rexp}(i s), z_{c}(s)=a s+d, p_{0}(s)=0, p_{1}(s)=\sigma .
$$

The singular integral equation (15), together with the condition of singlevaluedness of displacements (17), was solved by using the numerical technique proposed in the previous section. In Table I we present the dimensionless values of the stress intensity factors $\mathrm{K}_{\mathrm{A}} /\left(\sigma \mathrm{a}^{\frac{1}{2}}\right)$ and $\mathrm{K}_{\mathrm{B}} /\left(\sigma \mathrm{a}^{\frac{1}{2}}\right)$ at the crack tips $\mathrm{A}$ and $\mathrm{B}$ (Fig.3), for the case when $a / d=2.4$ and $d / R=0.2$, for several values of the numbers $\mathrm{n}_{0}$ and $\mathrm{n}_{\mathrm{c}}$ of the abscissae used. These results were obtained for $\mathrm{C}_{1}=\mathrm{C}_{2}=1$ and $z_{0}=c=0.5 i$. The influence of these constants on the values of the stress intensity factors $K_{A}$ and $K_{B}$ is not great. Moreover, from the results of Table $I$ we see that, as the numbers $n_{0}$ and $n_{c}$ of abscissae used increase, the values of the numerical results obtained converge. Finally, the values for $\mathrm{K}_{A} /\left(\sigma \mathrm{a}^{\frac{1}{2}}\right)$ and $\mathrm{K}_{\mathrm{B}} /\left(\sigma \mathrm{a}^{\frac{1}{2}}\right)$ presented Table I were seen to be in accordance with expected values of these factors presented in reference [21] for the same geometry and loading conditions (Table 3.3.1). 


\section{CONCLUSIONS}

By using the method of complex potentials of Kolosov and Muskhelishvili it was seen that every plane elasticity crack problem inside a finite or infinite medium with or without holes can easily be reduced to a complex Cauchy-type singular integral equation along both the cracks and the boundaries of the medium. These singular integral equations can be effectively solved by reducing them to systems of linear equations. In this way, it is possible to determine numerically the values of the stress intensity factors at crack tips for almost any geometry of the crack and the whole elastic medium and under arbitrary loading conditions on the cracks and the boundaries of the medium.

Even the case when the loading distributions are not the same on both edges of the cracks and there exists a resultant force, as well as a resultant moment on each crack can be treated.

As regards the accuracy of the numerical results obtained, this can be made as good as we want at the expense of computer time and the use of appropriate numerical integration rules.

\section{REFERENCES}

1. N.I. Muskhelishvili, Some Basic Problems of the Mathematical Theory of Elasticity, 4th Edn. P. Noordhoff, Groningen (1963).

2. R.V. Gol'dshteyn and R.L. Salganik, Planar problem of curvilinear cracks in an elastic solid. Mech. Solids 5, 54 (1970).

3. R.V. Gol'dshtein and L.N. Savona, Determination of crack opening stress intensity coefficients for a smooth curvilinear crack in an elastic plane. Mech. Solids 7, 64 (1972).

4. R.V. Gol'dstein and R.L. Salganik, Brittle fracture of solids with arbitrary cracks. Int. J. Fract. 10, 507 (1974). 
5. A.M. Lin'kov, Integral equations in the theory of elasticity for a plane with cuts, loaded by a balanced system of forces. Sov. Phys. Dokl. 19, 718 (1975).

6. V.V. Panasyuk, M.P. Savruk and A.P. Datsyshyn, A general method of solution of two-dimensional problems in the theory of cracks. Engng. Fract. Mech. 9, 481 (1977).

7. N.I. Ioakimidis and P.S. Theocaris, Array of periodic curvilinear cracks in an infinite isotropic medium. Acta Mech. 28, 239 (1977).

8. P.S. Theocaris and N.I. Ioakimidis, A star-shaped array of curvilinears cracks in an infinite isotropic elastic medium. J. Appl. Mech. 44, 619 (1977).

9. N.I. Ioakimidis and P.S. Theocaris, Doubly-periodic array of cracks in an infinite isotropic medium. J. Elast. 8, 157 (1978).

10. P.S. Theocaris and N.I. Ioakimidis, The problem of interaction between a misfitting inclusion and a crack in an infinite elastic medium. J. Elast. 9, 97 (1979).

11. N.I. Ioakimidis and P.S. Theocaris, On a method of numerical solution of a plane elasticity problem. Strojn. Cas. 29, 448, (1978).

12. P.S. Theocaris and N.I. Ioakimidis, Numerical Integration methods for the solution of Singular integral equations. Quart. Appl. Math. 35, 173 (1977).

13. P.S. Theocaris and N.I. Ioakimidis, The Inclusion problem in plane elasticity. Quart. J. Mech. Appl. Math. 30, 437 (1977).

14. P.S. Theocaris and N.I. Ioakimidis, The symmetrically branched crack in an infinite elastic medium. Zeit. Ang. Math. Phys. (ZAMP) 27, 801 (1976).

15. P.S. Theocaris and N.I. Ioakimidis, A method of solution of the problem of the unsymmetric cruciform crack in an infinite plane isotropic elastic medium. Acta Mech. 29, 127 (1978).

16. N.I. Ioakimidis and P.S. Theocaris, A system of curvilinear cracks in an isotropic elastic half-plane. Int. J. Fract. 15, No.4, 299 (1979). 
17. P.S. Theocaris and N.I. Ioakimidis, Stress intensity factors at crack tip near boundaries or other geometrical discontinuities. Int. J. Fract. 15 No 5, 419 (1979).

18. N.I. Ioakimidis and P.S. Theocaris, The Gauss-Hermite numerical integration method for the solution of the plane elastic problem of semi-infinite periodic cracks. Int. J. Engng Sci. 15, 271 (1977).

19. N.I. Ioakimidis and P.S. Theocaris, The practical evaluation of stress intensity factors at semi-infinite crack tips. Engng Fract. Mech. (to appear).

20. N.I. Ioakimidis and P.S. Theocaris, The numerical evaluation of a class of generalized stress intensity factors by use of the Lobatto-Jacobi numerical integration rule. Int. J. Fract. 14, 469 (1978).

21. D.P. Rooke and D.J. Cartwright, Compendium of Stress Intensity Factors, 1st Edn. Her Majesty's Stationary Office, London (1976).

\section{TABLE I}

Dimensionless values of the stress intensity factors $\mathrm{K}_{\mathrm{A}} /\left(\sigma a^{\frac{1}{2}}\right)$ and $\mathrm{K}_{\mathrm{B}} /\left(\sigma a^{\frac{1}{2}}\right)$ at the tips of the crack of Fig. 3 (for $a / d=2.4$ and $d / R=0.2$ ) obtained by using the numerical technique of this paper (with $C_{1}=C_{2}=1$ and $z_{0}=c=0.5 i$ ) for several values of $n_{0}$ and $n_{c}$.

\begin{tabular}{lccc}
\hline $\mathrm{n}_{0}$ & $\mathrm{n}_{\mathrm{c}}$ & $\mathrm{K}_{\mathrm{Z}} /\left(\sigma \mathrm{a}^{\frac{1}{2}}\right)$ & $\mathrm{K}_{\mathrm{B}} /\left(\sigma \mathrm{a}^{\frac{1}{2}}\right)$ \\
\hline 10 & 5 & 1.3269 & 1.3800 \\
15 & 5 & 1.3495 & 1.4321 \\
20 & 5 & 1.3542 & 1.4490 \\
25 & 5 & 1.3552 & 1.4529 \\
10 & 10 & 1.3265 & 1.3798 \\
15 & 10 & 1.3497 & 1.4322 \\
20 & 10 & 1.3544 & 1.4492 \\
\hline
\end{tabular}




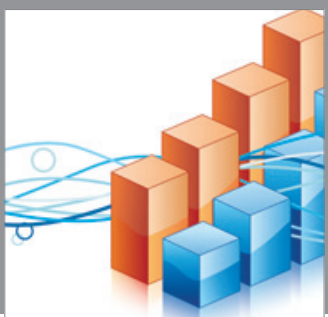

Advances in

Operations Research

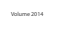

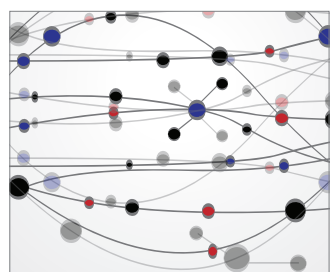

\section{The Scientific} World Journal
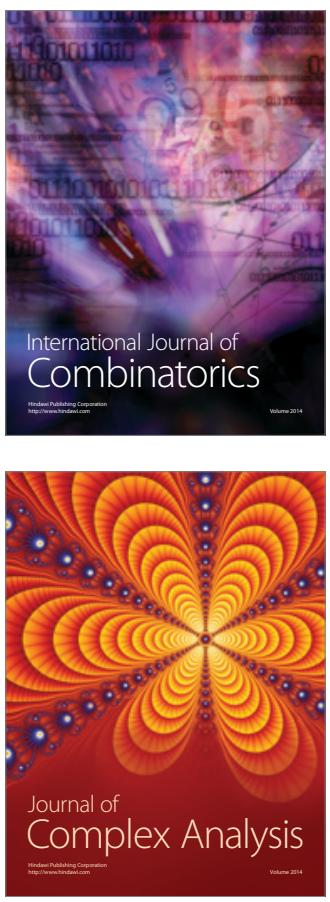

International Journal of

Mathematics and

Mathematical

Sciences
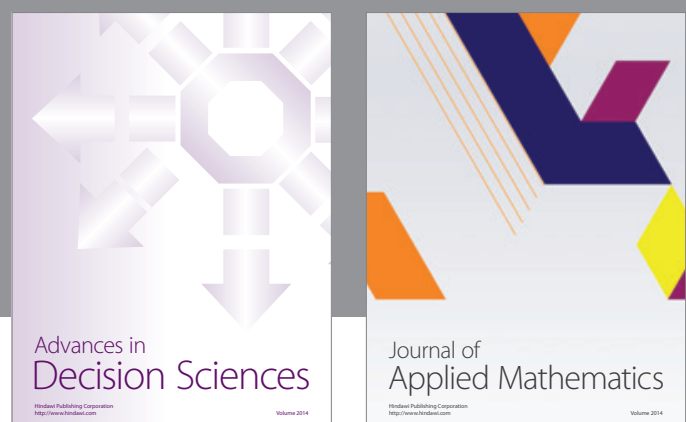

Journal of

Applied Mathematics
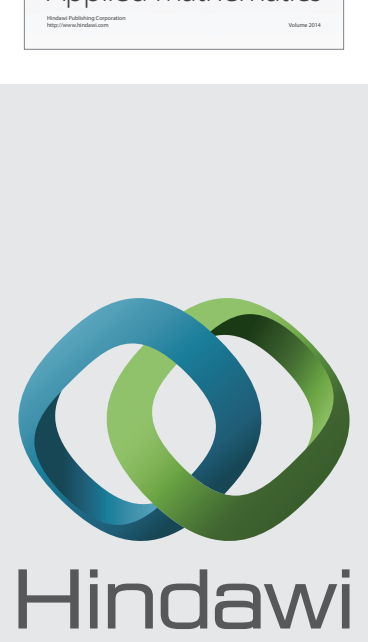

Submit your manuscripts at http://www.hindawi.com
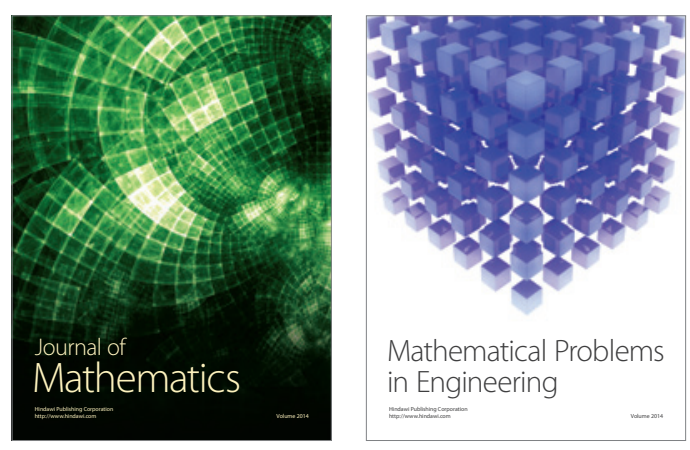

Mathematical Problems in Engineering
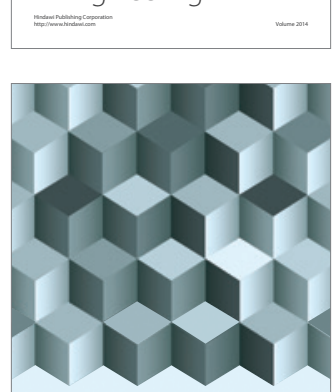

Journal of

Function Spaces
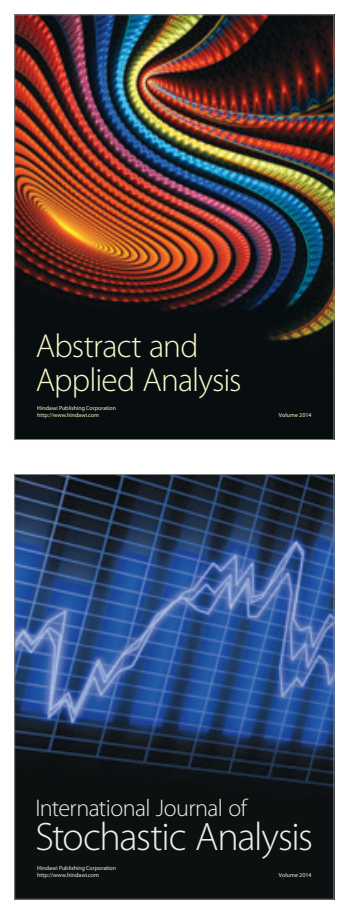

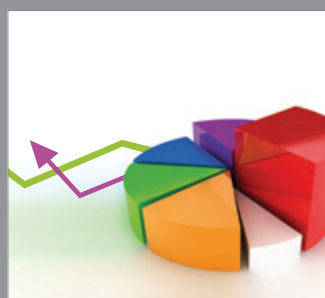

ournal of

Probability and Statistics

Promensencen
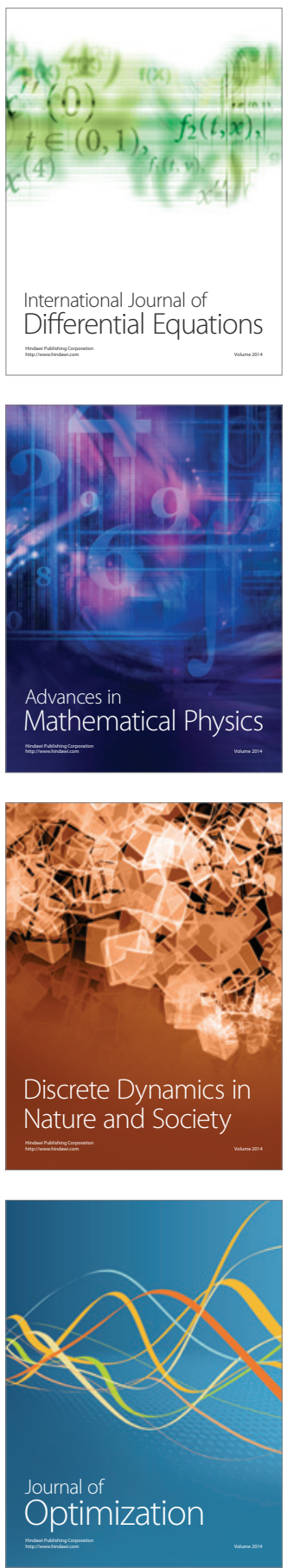